\title{
Taxonomic implications of morphometric analysis of earless seal limb bones
}

\author{
Morgan Churchill and Mark D. Uhen \\ Acta Palaeontologica Polonica 64 (2), 2019: 213-230 doi:https://doi.org/10.4202/app.00607.2019
}

Fossil Phocidae (earless seals) are mostly known from isolated postcranial material, forcing researchers to rely upon humeri and femora for the diagnosis of taxa and reconstruction of phylogeny. However, the utility of these elements has never been rigorously tested. Here, we provide the first quantitative analysis of morphometric data from the humerus and femur, incorporating measurement data from all extant genera as well as several fossil taxa. Principle components analysis (PCA) found that genera clustered together on PC1 and PC2, although there was poor segregation of taxa and extensive overlap with genera in adjacent regions of the morphospace. Discriminant function analysis (DFA) was able to sort fossil taxa into different subfamilies, but performed poorly at lower taxonomic levels. A preliminary review of phylogenetic characters found that while some characters performed well at distinguishing different subfamilies, many characters were poorly defined and not quantified, possessed greater individual variation than past studies suggested, or were more variable in fossil taxa. Our analyses suggest that the utility of isolated humeri and femora for diagnosis of new taxa has been greatly exaggerated, and that extreme caution should be applied to interpretations of taxonomy of fossil material based on isolated elements. Future research should instead focus on study of associated skeletons and cranial material. A thorough revision of fossil phocid taxonomy is needed, and many described taxa are likely to be nomina dubia and of limited use in phylogenetic analysis.

Key words: Mammalia, Phocidae, Monachinae, Pinnipedia, morphometrics, taxonomy, femur, humerus.

Morgan Churchill [churchim@uwosh.edu], Department of Biology, University of Wisconsin, 800 Algoma Blvd., Oshkosh WI 54901, USA. Mark D. Uhen [muhen@ gmu.edu], Department of Atmospheric, Oceanic, and Earth Sciences, George Mason University, 4400 University Dr., Fairfax, VA 22030, USA.

This is an open-access article distributed under the terms of the Creative Commons Attribution License (for details please see creativecommons.org), which permits unrestricted use, 
distribution, and reproduction in any medium, provided the original author and source are credited.

Faris Full text $(283.3 \mathrm{kB})$ ।

Far Supplementary file $(115.5 \mathrm{kB})$ 